\title{
Fasciculations in motor neuron disease: discharge rate reflects extent and recency of collateral sprouting
}

\author{
MARTIN JANKO, JOŽE V TRONTELJ, KSENIJA GERŠAK \\ From the University Institute of Clinical Neurophysiology, University Medical Centre of Ljubljana, Yugoslavia
}

SUMMARY Single fibre EMG recordings were made from 152 fasciculating motor units in 17 patients with motor neuron disease. All recordings showed abnormal jitter, many $(75 \%)$ displayed intermittent blocking, and most had abnormal fibre density (mean 4.3), demonstrating considerable degrees of collateral sprouting supported by the fasciculating motor units, and varying degrees of functional immaturity of the new axonal twigs and the motor end plates. The SFEMG abnormalities reflecting both the degree and the recency of collateral reinnervation correlated with the mean interdischarge interval, suggesting that the properties of the generator site depend on the functional state of the fasciculating motor unit as a whole.

Fasciculations are considered to represent involuntary activity of motor units; ${ }^{1}$ they may be an important early sign of motor neuron disease and have therefore attracted the considerable interest of neurologists and neurophysiologists. Differentiation between these "malignant" and the commonplace "benign" fasciculations of healthy people remains uncertain, although some differences have been described. ${ }^{2-4}$ One of the differences is in the discharge rate, which is higher in the case of the "benign" fasciculations. ${ }^{4}$ The site of generation has been investigated by several authors. ${ }^{256-9}$ The prevailing opinion holds that most, if not all, fasciculations are generated distally in the axonal tree, ${ }^{78}$ perhaps as distal as the presynaptic portion of the motor end plate. ${ }^{8}$ Some single fibre observations supported distal generator sites and drew attention to differences between fasciculations in motor neuron disease and those in healthy persons. The latter may not represent activity of individual motor units but may be based on ephaptic activation of a bundle of adjacent muscle fibres, driven by an irregularly fibrillating muscle fibre. - $^{-11}$

This study was undertaken with the aim of quantifying certain parameters of fasciculations in motorneuron disease, particularly their repetition rates, and to observe some morphological and functional characteristics of the fasciculating motor units, such as fibre

Correspondence to: Professor Martin Janko, University Institute of Clinical Neurophysiology, University Medical Centre, Zaloska 7, 61105 Ljubljana, Yugoslavia.

Received 8 December 1988 and in revised form 12 June 1989 Accepted 17 July 1989 density, degree of abnormal jitter and blocking, degree of asynchronicity of the participating muscle fibres and the availability of these motor units for voluntary or electrical activation. An attempt was made to correlate the above parameters to firing characteristics of the fasciculating motor units. Part of the data has already been reported. ${ }^{12}$

\section{Methods and patients}

A single fibre EMG electrode was inserted into a muscle showing fasciculations and its position was adjusted to obtain recordings containing single fibre action potentials of sufficiently high amplitude $(>200 \mathrm{uV})$ and short rise time $(<0.3 \mathrm{~ms})$, which are the usual criteria to recognise them as being generated by individual muscle fibres. All the recorded fasciculation potentials contained at least two and up to 12 single fibre components.

The fasciculation potentials were recorded by a Medelec MS-6 electromyograph, stored on magnetic tape and photographed on a UV sensitive film in raster mode for subsequent analysis, which included identification of the individual fasciculating motor units, the number of single fibre components in the complex potentials, estimation of the jitter between the individual components as well as identification of intermittent blocking when present, and finally, measurement of interdischarge intervals. We decided to use manual time measurement from these recordings rather than the computerised methods, as it allowed reliable identification not only of the individual fibre components but also of the studied motor unit when more than one appeared in the same recording. Occasionally this was not easy, especially with fasciculation potentials of greatly variable shape. The resolution of time measurement was $10-50 \mu$ s for the jitter and $50 \mathrm{~ms}$ for the interdischarge intervals. Twenty to fifty successive discharges of any fasciculating motor unit were 
analysed in this way. Acquisition of longer series of discharges according to the usual methodological standards of jitter measurement in voluntarily activated muscle proved impractical, as it would take too long due to the low mean discharge rate of the fasciculation potentials.

In 10 fasciculations with complex, rather stable and well recognisable shapes, recorded in muscles with fairly good voluntary power, attempts were made to recruit the fasciculating motor unit in voluntary contraction.

In the same cases, needle stimulating electrodes (a pair of Teca MF 37 monopolar Teflon coated tungsten wire electrodes with bare tips) were introduced about $2 \mathrm{~cm}$ away from the recording electrode and their position was adjusted so that the fasciculating potential was then driven by the electrical stimulus (rectangular pulse $0.05 \mathrm{~ms}$, up to $50 \mathrm{~V}$, 2-5 Hz).

Included in the study were 17 patients with motor neuron disease, of whom seven had nearly pure spinal form, seven had classical amyotrophic lateral sclerosis, and the remaining three had bulbar form of the disease. Their ages were 43-74 years, mean 66 years. They were diagnosed according to standard clinical and electromyographic criteria, and other diseases such as spondylotic myelopathy were carefully excluded. None of the patients had clinical or neurophysiological evidence suggesting superimposed radiculopathy or peripheral neuropathy. The patients were in different stages of their disease, including two in the terminal stage with nearly complete tetraplegia.
The muscles selected for the study were usually those in which fasciculations were readily visible. They included the forearm flexors and extensors, the deltoid, and the tibialis anterior. Some of these had nearly normal strength while others showed different degrees of weakness or even complete paralysis.

\section{Results}

In total, 152 fasciculation potentials were recorded and analysed. Of these, $38(25 \%)$ showed no blocking of the individual single fibre components; they are referred to hereafter as "stable fasciculation potentials". In a further 87 fasciculation potentials (57\%), one to three components showed intermittent blocking (mean 10\% blocking in 20 discharges). These were termed "moderately unstable fasciculation potentials". c The remaining 27 fasciculation potentials $(18 \%) \overrightarrow{0}$ exhibited an extreme degree of blocking of nearly all components which made their identification and coun- $\vec{\omega}$ ting difficult. They were classified as "highly unstable fasciculation potentials"'(fig 1).

The jitter was analysed in 312 single fibre compon- or ents (fig 2). Even in the group of stable fasciculation potentials, about one half of the values exceeded the $\vec{N}$ upper normal limit of neuromuscular jitter in volun $\vec{\omega}$
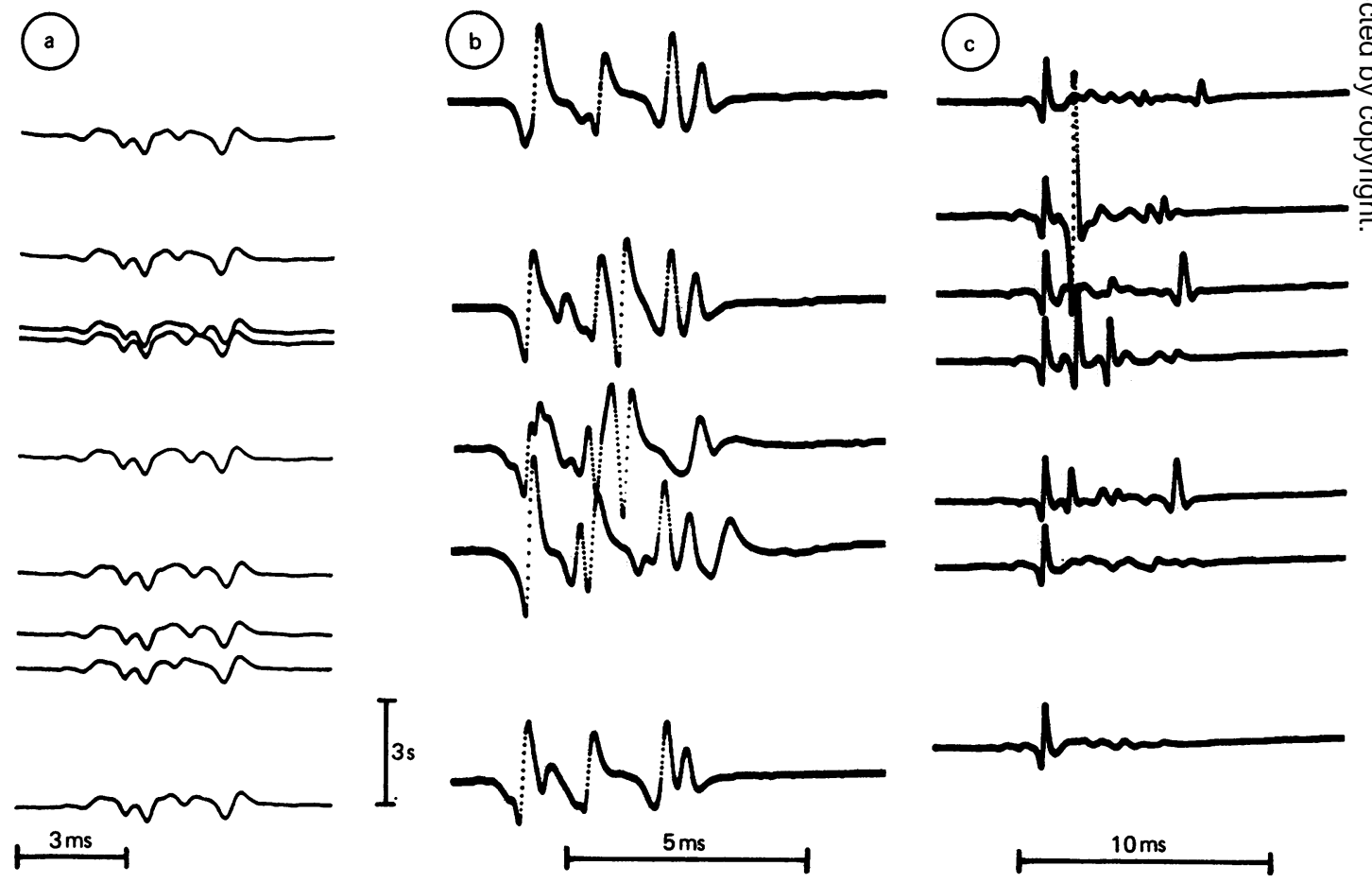

Fig 1 Examples of relatively stable (A), moderately unstable (B), and highly unstable (C) fasciculation potentials, as recorded by single fibre EMG. 


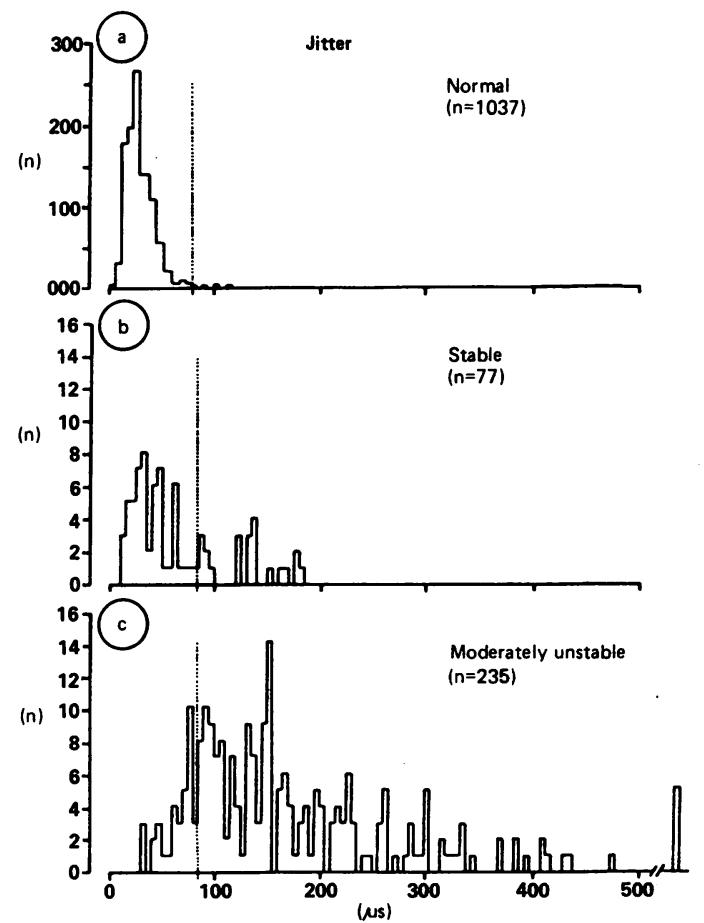

Fig 2 Jitter values (expressed as $M C D$ measured in the group of stable $(B)$ and moderately unstable fasciculation potentials $(C)$, compared to combined normal values for the biceps brachii, extensor digitorum communis and tibialis anterior ( $A$, from reference ${ }^{10}$ ).

tarily activated motor units of healthy people. Naturally, the jitter could not be precisely estimated in the group of highly unstable fasciculation potentials.

The number of single fibre action potentials that could be identified in the fasciculation potential according to the criteria used in fibre density estimation ${ }^{10}$ was the lowest in stable fasciculation potentials and the highest in the highly unstable ones (fig 3). The mean (SD) fibre densities calculated for each of the three groups were $3.05(1.02), 3.79(1.51)$, and 7.15 $(2 \cdot 36)$, respectively. The mean fibre density for all fasciculating motor units wäs 4.3.

The mean duration of the fasciculation potentials, as measured between the first and the last single fibre component, was $3.2 \mathrm{~ms}(1.8 \mathrm{~ms})$ for the stable group, $4.0 \mathrm{~ms}(2.6 \mathrm{~ms})$ for the moderately unstable group, and $12.2 \mathrm{~ms}(6.9 \mathrm{~ms})$ for the highly unstable group, respectively. The mean duration for the whole material was $5 \cdot 25 \mathrm{~ms}$.

The repetition rates were quite irregular in all cases. Apart from the wide, approximately random, scattering of consecutive interdischarge intervals in each fasciculating motor unit, there were many cases of distinct short intervals, below $300 \mathrm{~ms}$ (figs 1A and 6). These were considered to represent extra-discharges ${ }^{11}$ and were not included in the computed mean values. The mean (SE) interdischarge intervals were $2.88 \mathrm{~s}$ $(0.36 \mathrm{~s})$ for the stable fasciculation potentials, $5.35 \mathrm{~s}$ $(0.72 \mathrm{~s})$ for the moderately unstable group, and $10.44 \mathrm{~s}$

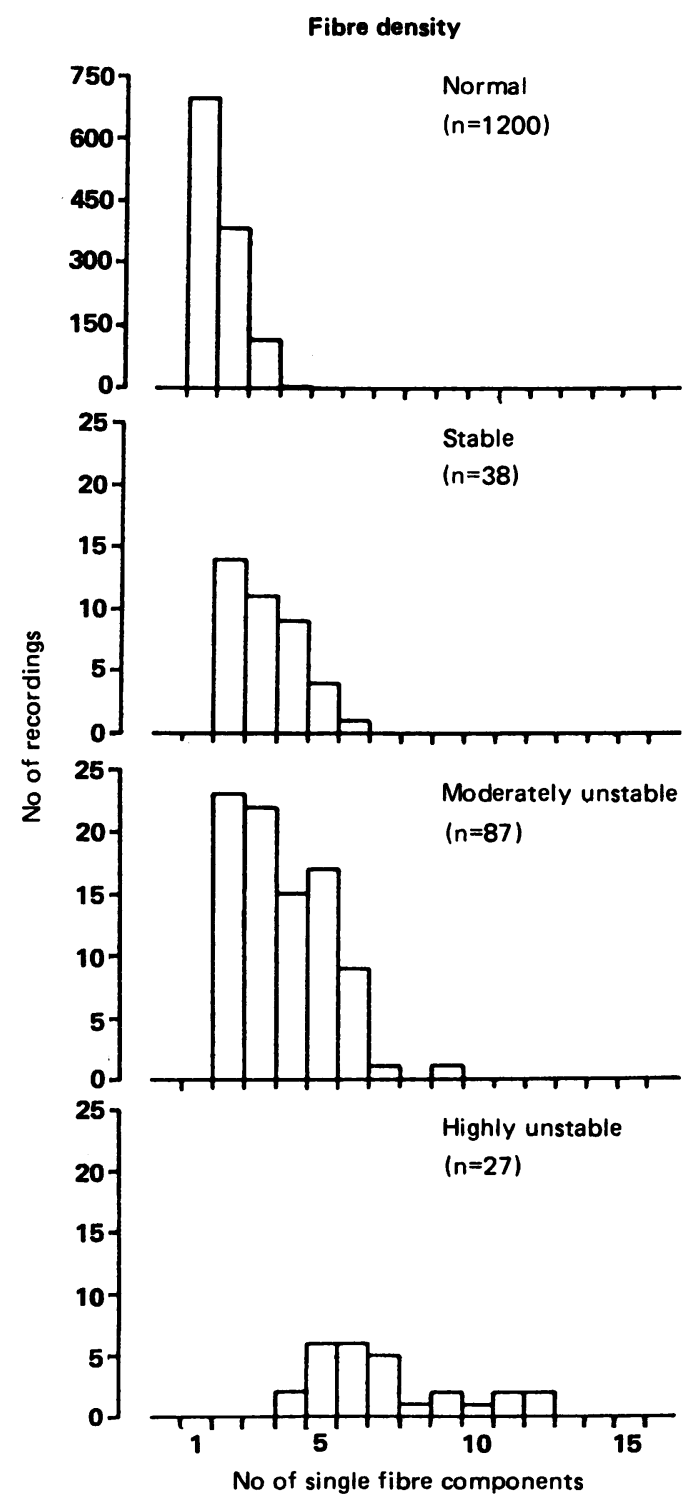

Fig 3 The number of single muscle fibre components (fibre density) in the three groups of fasciculation potentials, in comparison to normal fibre density in voluntarily activated motor units of the etensor digitorum communis and tibialis anterior muscles (the normal material is from reference ${ }^{10}$ ). 


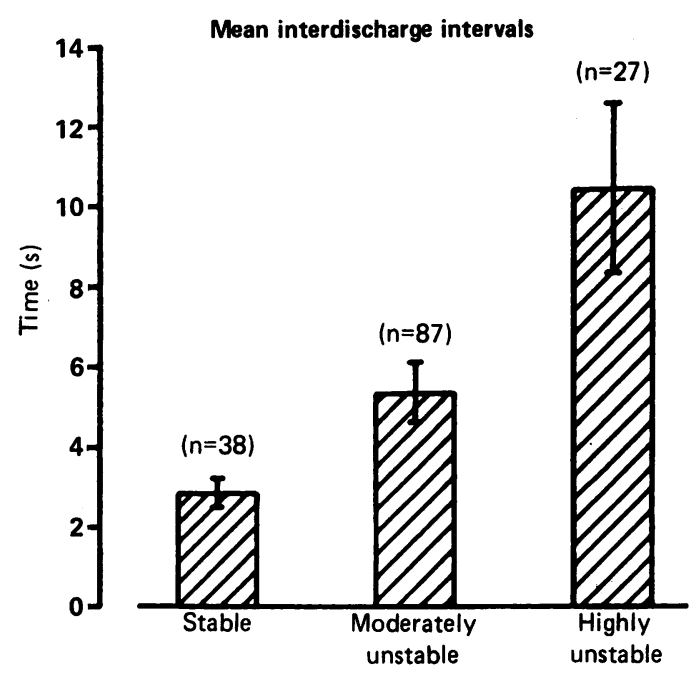

Fig 4 The different mean inter-discharge intervals in the three groups of fasciculation (A stable, B moderately unstable, $C$ highly unstable fasciculations.

$(2.43 \mathrm{~s})$ for the highly unstable group (fig 4). The overall mean repetition rate was $0 \cdot 17 \mathrm{~Hz}$.

The attempts at voluntary recruitment of the fasciculating motor units failed in all cases, despite considerable degrees of force of voluntary contraction.

On the other hand, it was possible to drive the same fasciculating motor units by intramuscular electrical stimulation in all of the ten cases where tried. The complex action potential elicited by electrical stimulation not only contained the same single fibre components as the fasciculation potential, but also displayed the same temporal relationship between the individual components and had the same total duration (fig 5).

The temporal order of spikes remained unchanged throughout the period of observation in the stable and moderately unstable fasciculation potentials, except in one case, where intermittent reversal of sequence of two spikes was seen to occur (fig 6). The highly unstable fasciculations showed frequent changes in the order of spikes which could, however, be explained by the very large jitter.

No significant correlation could be detected between the clinical type of the disorder, its stage, rate of progression, or weakness of the muscle studied and the frequency of each of the three types of fasciculation potentials and their properties. However, the highly unstable type tended to be more common in terminal cases or in very weak muscles.

\section{Discussion}

The method of single fibre EMG was selected for thio study because it allows a more reliable identification of the fasciculating motor units when compared, foro example, to concentric needle EMG. Furthermore, 传 gives a more detailed insight into morphologicat parameters such as the fibre density, as well as functional parameters, such as reliability of. neuromuscular transmission (reflected in jitter an blocking), degree of firing synchronicity (total dura? tion of the fasciculation potential), and occasionally information regarding the site of origin (reversin 5 order of single fibre components suggests alternation between different sites, at least some of which must be् quite distal, in the axonal branches).

Source of fasciculations It is generally believed tha $\vec{b}$

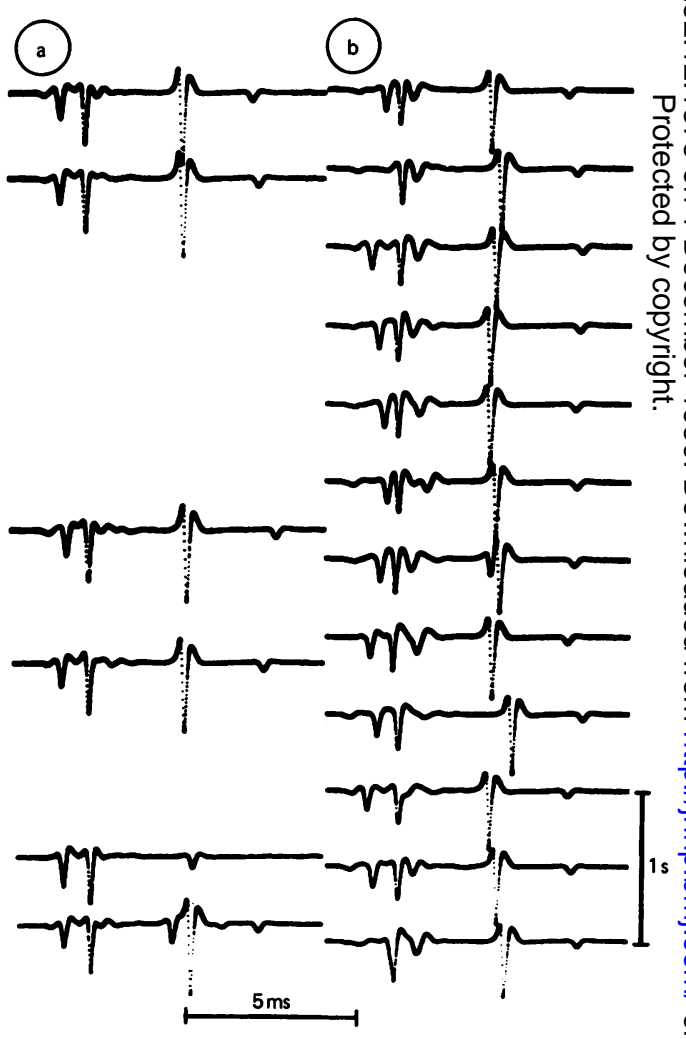

Fig 5 Fasciculating motor unit $(A)$, subsequently driven by electrical stimulation of its motor axon $(B)$. Note the unchanged configuration of the complex potential. With either mode of activation, some components block intermittently. 

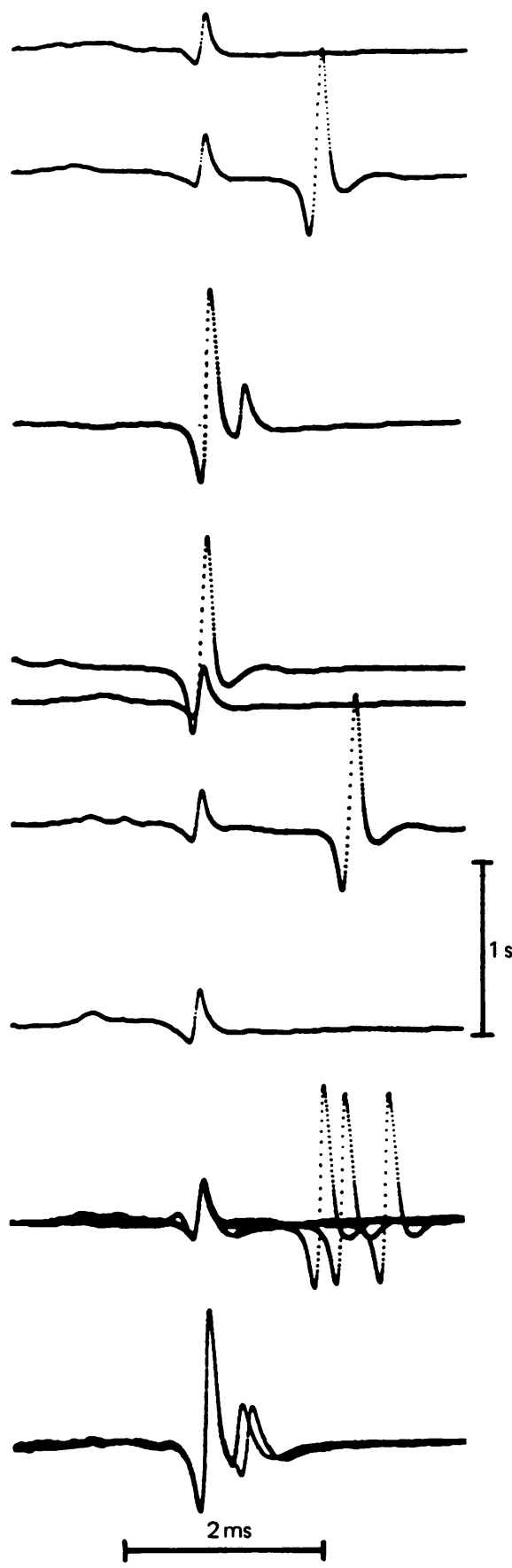

Fig 6 A fasciculation potential represented in the recording with two muscle fibres, appearing independently or jointly with variable interpotential intervals and in changing order. Top traces with moving film, bottom two superimposed recordings. fasciculations in motor neuron disease represent spontaneous discharges of individual motor units. Perfect proof would be to record the same discharge in voluntary activity. This was done in the study by Conradi and Grimby, ${ }^{8}$ and Conradi et al ${ }^{13}$ whose ALS patients were able to drive their fasciculating motor units voluntarily every time this was tried. Similar attempts, however, were unsuccessful in the study by Trojaborg and Buchthal, ${ }^{4}$ as well as this study. On the other hand, electrical stimulation of the distal part of the motor axon which exactly reproduced the shape of the fasciculation can also be regarded as strong evidence in support of the motor unit as the generator of fasciculations. This may not be the case with benign fasciculations which appear to be based on ephaptic activation of adjacent muscle fibres by irregularly fibrillating fibres, ${ }^{9-11}$ similar to open loop complex repetitive discharges. ${ }^{14}$ Some characteristics of benign fasciculations can clearly be reproduced by direct electrical stimulation of muscle fibres (Trontelj et al in preparation).

Site of impulse initiation Experiments with nerve blocks in patients with motor neuron disease ${ }^{15}$ have shown that fasciculations are perhaps diminished but not abolished. Fasciculations persisted for 12-14 days after femoral nerve section in terminally ill patients with motor neuron disease. ${ }^{7}$ This would seem to place the site of their generation distally in the motor unit, although a proportion may originate from proximal segments, perhaps including the ventral horn cell itself. Wettstein, ${ }^{5}$ using collision technique, and Roth, ${ }^{6}$ using F-responses, were able to identify quite distal generation in most of the studied fasciculating motor units. Conradi and associates ${ }^{813}$ suggested that the impulses may be triggered from the presynaptic acetylcholine receptors in the end plate region.

When there is more than a single site of impulse generation, this may be seen in single fibre EMG recordings, provided that the sites are quite distal, for example, in the terminal axonal branches. In this case the individual components in the multiple fibre potential may alternate between two or several positions, and even the order of the components may be reversed. Such reversal has been domonstrated in extra-discharges, ${ }^{10}$ but was seen in only two out of 125 stable and moderately unstable fasciculation potentials in this study. It was often observed in highly unstable fasciculation potentials. This could not be attributed with certainty to alternating sites of generation, as it could simply be due to the extremely large motor end plate (and possibly axonal) jitter in these cases. The constant order of the individual components does not exclude the possibility that the impulses start at the motor end plate, since alternation is not seen if the impulses are consistently triggered from only a single rather than multiple sites. Experiments with intramuscular 
stimulation of the presumably preterminal portions of the motor axons, which exactly reproduced the order and the temporal relationships of the different single fibre components, seem to be also compatible with more proximal generation sites, for example, at some branching points of the axons, or at the points of newly formed collateral sprouts, or even up to the ventral horn cell itself.

If the impulses start distally in the terminal axonal twigs, the motor unit is activated after antidromic conduction and via the axon reflexes. This results in an increased duration of the fasciculation potential compared to the action potential of the same motor unit when activated in the usual orthodromic way. Unfortunately, attempts at voluntary activation and more proximal nerve stimulation which would clarify the issue failed.

Properties of the fasciculating motor units The apparent unavailability of the fasciculating motor units for voluntary activation (only tested adequately in 10 cases) might suggest that their ventral horn cells are functionally altered, having increased thresholds for voluntary recruitment, while some may be degenerating or even dead. A less likely explanation, in our cases, would be the loss of voluntary control due to upper motor neuron degeneration. The patients in whom the test was made had nearly normal voluntary power of the muscle tested, and had very mild or absent pyramidal signs. Another possible explanation could be that the fasciculating (presumably sick) motor units belong to those that normally have high threshold for voluntary recruitment, higher degrees of voluntary contraction at which selectivity of recording could be lost were not used. Conradi et a ${ }^{13}$ used the extensor digitorum brevis muscle with presumably fewer surviving motor units (due both to normal and disease-related drop out) which were easier to observe in isolation even at higher degrees of voluntary effort.

None of the fasciculating motor units in this study had normal single fibre EMG parameters. Most of them had high fibre density, the mean value being 3.98 , compared to the upper normal limit of 1.99 in the extensor digitorum communis or 2.07 in the tibialis anterior. ${ }^{10}$ All had at least moderately abnormal jitter, and a majority showed intermittent blocking of varying numbers of single fibre components.

It proved convenient to divide all fasciculation potentials into three groups, according to the degree of abnormality in impulse transmission in the terminal axonal twigs and across the motor end plates. The physiological relevance of such classification is that it reflects the amount of recent collateral reinnervation. ${ }^{10}$ The group with highly unstable fasciculation potentials is rather small in this material, but is considered to be under-represented. The reason is that such fasciculations were, due to their extreme variability, difficult to identify as being generated by the same ${ }_{\mathbb{O}}^{Z}$ group of muscle fibres, and many doubtful cases were discarded. This difficulty could not possibly be obviated, particularly in profusely fasciculating mus- $\frac{\bar{A}}{0}$ cles. Nevertheless, it is felt that the exclusion of the 0 dubious recordings did not essentially change the results.

These single fibre EMG abnormalities reflect $\mathrm{a} \stackrel{\overline{0}}{\Rightarrow}$ considerable degree of collateral axonal sprouting to reinnervate muscle fibres of the degenerated ventral $\overrightarrow{\vec{A}}$ horn cells. Whether it is collateral sprouting under $\frac{\text { ? }}{0}$ certain conditions which makes the motor unit suscep- $\frac{C}{0}$ tible to fasciculation, is an interesting possibility which $\frac{\bar{D}}{\bar{D}}$ is difficult to test.

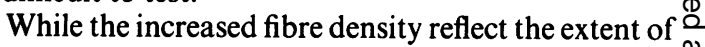
collateral reinnervation, the abnormal jitter and on blocking suggest that the reinnervation has taken $\overrightarrow{0}$ place relatively recently. In a neurologically healthy individual with a partial nerve injury followed by $\vec{\omega}$ collateral reinnervation, most of the initially abnormal jitter and blocking will disappear after about six months. ${ }^{10}$ Whether such functional maturity is ever or reached in motor neuron disease is not known. As denervation progresses and collateral reinnervation $\vec{N}$ continues, the growing motor units always show $\vec{\nabla} \vec{\omega}$ degree of abnormal jitter and blocking, at least in $v$ part of their territories. Whether some of the abnorma $\mathrm{O}$ jitter and blocking is in fact due to degeneration of the original motor end plates rather than reinnervation unknown. Denervation itself, that is death of the ventral horn cell per se, is unlikely to be the cause Virtually all motor end-plates tested in the acute stage after neurotmesis but before Wallerian degeneratio in neurologically normal individuals showed normal $\vec{\theta}$ jitter on electrical stimulation of the cut axons, at least $\subseteq$ at rates of $1 \mathrm{~Hz}$ or below, which is comparable to the discharge rate of fasciculating motor units (Trontelj $e t$ al, unpublished material).

It might be of interest to compare the SFEMG parameters of the fasciculating motor units to those recruited in voluntary activity. This was not done in the muscle that was studied, although each patient had a routine SFEMG study in the extensor digitorum communis.

The interdischarge intervals (IDI) were quite irregular in all of the studied fasciculations, as is known from previous studies and clinical experience. Yet the degree of irregularity was significantly higher in the group of highly unstable fasciculations. As mentioned above, there was a distinct class of low IDI values below $300 \mathrm{~ms}$ which was considered to represent extra-discharges, generated during the period of supernormal excitability of the pacemaker site. These were excluded when computing the mean IDI values, as well as the IDI variability. Interestingly, the interval to the extra-discharge was in general 
considerably longer than in the case of extra-discharges following voluntary discharges in myopathies or in some neurogenic conditions where most of the values fall between 3.5 and $15 \mathrm{~ms} .{ }^{11}$ This may reflect a different time course of supernormality of the generator site in the axon (or at the motor end plate.) Conceivably, there may be a single generator site for both the primary fasciculation and its extradischarge, or the extra-discharge is triggered from another site. In two recordings, one of which is illustrated in fig 6 , a reversed order of single fibre components suggested that the fasciculation occasionally originated from a second generator site and that at least one of the two was situated rather peripherally.

The IDI irregularity of fasciculations in motor neuron disease as seen in this study was larger than that of spontaneous discharges in some other neurogenic conditions, for example, neuro-myotonia, ${ }^{\prime \prime}$ or entrapment neuropathies where the spontaneous discharges may often be rather regular. ${ }^{16}$

The mean IDI, computed for all fasciculations of all the three groups, was $5.0 \mathrm{~s}$, which is longer than that reported by Denny-Brown and Pennybacker,' as well as Trojaborg and Buchthal, ${ }^{4}$ at least in part because of the exclusion of the IDI values below $0 \cdot 3 \mathrm{~s}$ in this study. Another reason might be a more selective recording used in this study, better suited to distinguish between similar action potentials of two different motor units.

This study demonstrated clear cut correlations between the degree of instability and duration of the fasciculation potentials on the one hand, and the fibre density of the fasciculating motor units on the other. That is, between parameters reflecting the recency and the extent of collateral sprouting (the former two parameters may also be related to processes of motor unit degeneration). Alternatively, all these parameters were directly correlated to the mean IDI. In other words, those fasciculating units with high fibre density, long potential duration and high degree of instability discharged at significantly lower mean rates, approximately 2.5 times lower than those with less increased fibre density, shorter potential duration and relatively stable impulse transmission. This seems to indicate that both the amount and recency of collateral sprouting supported by the fasciculating motor unit is reflected in the properties of the generator site.

\section{References}

1 Denny-Brown D, Pennybacker JB. Fibrillation and fasciculation in voluntary muscle. Brain 1938;61:311-32.

2 Denny-Brown D. Clinical problems in neuromuscular physiology. Am J Med 1953;15:368-90.

3 Reed DM, Kurland LT. Muscle fasciculations in a healthy population. Arch Neurol 1963;9:363-7.

4 Trojaborg W, Buchthal F. Malignant and benign fasciculations. Acta Neurol Scand 1965;41, Suppl 13: 251-4.

5 Wettstein A. The origin of fasciculations in motoneurone disease. Ann Neurol 1979;5:295-300.

6 Roth G. Fasciculations and their F-response. J Neurol Sci 1984;63:299-306.

7 Forster FM, Borkowski WJ, Alpers BJ. Effect of denervation on fasciculations in human muscles. Arch Neurol Psychiat 1946;56:276-83.

8 Conradi S, Grimby L. Fasciculations in ALS: implications of possible origin from presynaptic $\mathrm{AcH}$ receptors in the end-plate region. Electroenceph clin Neurophysiol 1983;56:S61.

9 Trontelj JV, Stålberg E. Spontaneous activity within the motor unit. Electroenceph clin Neurophysiol 1977; 43:613-4.

10 Stålberg E, Trontelj JV. Single Fibre Electromyography. Old Woking, Surrey: Mirvale Press, 1979:1-244.

11 Stålberg E, Trontelj JV. Abnormal discharges generated within the motor unit as observed with single fibre electromyography. In: Culp JW, Ochoa J eds. Abnormal nerves and muscles as impulse generators. New York: Oxford University Press, 1982:443-74.

12 Trontelj JV, Janko M, Jenič K. Single fiber EMG of fasciculations in motor neuron disease. Electroenceph clin Neurophysiol 1987;66:S127.

13 Conradi S, Grimby L, Lundemo G. Pathophysiology of fasciculations in ALS as studied by electromyography of single motor units. Muscle Nerve 1982;5:202-8.

14 Trontelj JV, Stålberg E. Bizarre repetitive discharges recorded with single fibre EMG. J Neurol Neurosurg Psychiatry 1983;46:310-6.

15 Swank RL, Price JC. Fascicular muscle twitching in amyotrophic lateral sclerosis. Arch Neurol Psychiat 1943;49:22-6.

16 Spaans F. Spontaneous rhythmic motor unit potentials in the carpal tunnel syndrome. J Neurol Neurosurg Psychiatry 1982;45:19-28. 